\title{
Effects of cost surface uncertainty on current density estimates from circuit theory
}

\author{
Jeff Bowman ${ }^{\text {Corresp., } 1,2 \text {, Elizabeth Adey }}{ }^{2}$, Siow Yan Jennifer Angoh ${ }^{2}$, Jennifer Baici ${ }^{2}$, Michael GC Brown ${ }^{2}$, Chad \\ Cordes $^{1}$, Arthur Dupuis ${ }^{2}$, Sasha Newar ${ }^{2}$, Laura Scott ${ }^{2}$, Kirsten Solmundson ${ }^{2}$ \\ 1 Ontario Ministry of Natural Resources and Forestry, Peterborough, Canada \\ ${ }^{2}$ Trent University, Peterborough, Canada \\ Corresponding Author: Jeff Bowman \\ Email address: jeff.bowman@ontario.ca
}

Background Conservation practitioners are often interested in developing land use plans that increase landscape connectivity, which is defined as the degree to which the landscape facilitates or impedes movement among resource patches. Landscape connectivity is often estimated with a cost surface that indicates the varying costs experienced by an organism in moving across a landscape. True, or absolute costs are rarely known however, and therefore assigning costs to different landscape elements is often a challenge in creating cost surface maps. As such, we consider it important to understand the sensitivity of connectivity estimates to uncertainty in cost estimates. Methods We used simulated landscapes to test the sensitivity of current density estimates from circuit theory to varying relative cost values, fragmentation, and number of cost classes (i.e., thematic resolution). Current density is proportional to the probability of use during a random walk. Using Circuitscape software, we simulated electrical current between pairs of nodes to create current density maps. We then measured the correlation of the current density values across scenarios. Results In general, we found that cost values were highly correlated across scenarios with different cost weights (mean correlation ranged from 0.87 to 0.92 ). Changing the spatial configuration of landscape elements by varying the degree of fragmentation reduced correlation in current density across maps. We also found that correlations were more variable when the range of cost values in a map was high. Discussion The low sensitivity of current density estimates to relative costs weights suggests that the measure may be reliable for land use applications even when there is uncertainty about absolute cost values, provided that the user has the costs correctly ranked. This finding should facilitate the use of cost surfaces by conservation practitioners interested in estimating connectivity and planning linkages and corridors. 


\section{Effects of cost surface uncertainty on current density}

\section{2 estimates from circuit theory}

3

4

5

6

7

8

9

10

11

12

13

14 3Provincial Geomatics Centre, Ontario Ministry of Natural Resources and Forestry,

15 Peterborough, Ontario, Canada

16

17

18

19

20

21

22
Jeff Bowman ${ }^{1,2}$, Elizabeth Adey ${ }^{2}$, Siow Yan Jennifer Angoh² ${ }^{2}$ Jennifer E. Baici², Michael G.C. Brown $^{2}$, Chad Cordes ${ }^{3}$, Arthur E. Dupuis ${ }^{2}$, Sasha L. Newar ${ }^{2}$, Laura M. Scott ${ }^{2}$, Kirsten

Solmundson ${ }^{2}$

${ }^{1}$ Wildlife Research and Monitoring Section, Ontario Ministry of Natural Resources and Forestry, Peterborough, Ontario, Canada

${ }^{2}$ Environmental and Life Sciences Graduate Program, Trent University, Peterborough, Ontario, Canada

Corresponding Author:

Jeff Bowman ${ }^{1,2}$

Trent University DNA Building, 2140 East Bank Drive, Peterborough, ON, K9L 0G2

Email address: jeff.bowman@ontario.ca 


\section{Abstract}

24

\section{Background}

26 Conservation practitioners are often interested in developing land use plans that increase 27 landscape connectivity, which is defined as the degree to which the landscape facilitates or impedes movement among resource patches. Landscape connectivity is often estimated with a cost surface that indicates the varying costs experienced by an organism in moving across a landscape. True, or absolute costs are rarely known however, and therefore assigning costs to different landscape elements is often a challenge in creating cost surface maps. As such, we consider it important to understand the sensitivity of connectivity estimates to uncertainty in cost estimates.

\section{Methods}

We used simulated landscapes to test the sensitivity of current density estimates from circuit theory to varying relative cost values, fragmentation, and number of cost classes (i.e., thematic resolution). Current density is proportional to the probability of use during a random walk. Using Circuitscape software, we simulated electrical current between pairs of nodes to create current density maps. We then measured the correlation of the current density values across scenarios.

\section{Results}

In general, we found that cost values were highly correlated across scenarios with different cost weights (mean correlation ranged from 0.87 to 0.92 ). Changing the spatial configuration of landscape elements by varying the degree of fragmentation reduced correlation in current density across maps. We also found that correlations were more variable when the range of cost values in a map was high.

\section{Discussion}

The low sensitivity of current density estimates to relative costweights suggests that the measure may be reliable for land use applications even when there is uncertainty about absolute cost values, provided that the user has the costs correctly ranked. This finding should facilitate the use 
53 of cost surfaces by conservation practitioners interested in estimating connectivity and planning 54 linkages and corridors.

\section{Introduction}

57 Habitat loss and fragmentation can lead to increased extinction risk of populations by limiting 58 landscape connectivity, which is defined as the degree to which landscape structure facilitates or 59 impedes movement among resource patches (Taylor et al. 1993). Exploring the impacts of 60 changes in landscape connectivity is a significant conservation research focus, because

61 enhancements to connectivity have the potential to help mitigate extinction risk (Crooks et al.

62 2017). For example, the creation of a connected network of protected areas in India resulted in a $632385 \mathrm{~km}^{2}$ increase of critical habitat for tigers (Panthera tigris) (Gubbi et al. 2015).

64 In recent years, evaluating landscape connectivity has often been done through the use of a 65 cost surface, which is a 2-dimensional depiction of a landscape where the landscape elements are assigned relative costs that are meant to represent what organisms experience as they move

67 through the landscape (Koen et al. 2012). Typically, higher costs indicate higher risk, and therefore lower probability of moving successfully through the element. Cost surfaces are generally depicted as a raster of pixels, where each pixel is assigned a cost. Different movement models, such as circuit theory or least cost path analysis (Adriaensen et al. 2003, McRae 2006), are then used to estimate connectivity between locations based on the raster of cost values. Cost assignment is often driven by species-specific considerations, because connectivity is generally a species-specific construct (Taylor et al. 1993).

A challenge in developing accurate cost surfaces, and therefore in modelling connectivity, is uncertainty in assigning costs to different landscape elements. True costs are rarely known, and therefore a variety of methods are used to estimate them, including expert opinion, empirical 
77 movement data, and gene flow (Spear et al. 2010, Koen et al. 2012). The accuracy of cost values

78 may be evaluated through comparison with field data (Cushman et al. 2006, Lee-Yaw et al.

79 2009), but even in such cases, it is often the relative, rather than absolute costs that are learned.

80 Given uncertainty in cost values, it seems important to understand the sensitivity of different 81 connectivity estimates to variations in the estimated costs (Adriaensen et al. 2003). For example,

82 Rayfield et al. (2009) found that the sensitivity of placing least-cost routes is positively related to 83 the amount of habitat fragmentation and inversely related to the hospitality of the matrix. Beier,

84 Majka, \& Newell (2009) demonstrated that if a model has the correct rank order of costs, the 85 placement of a least-cost path is robust to uncertainty. They recommended that users undertake sensitivity analyses when developing cost surfaces. Koen et al. (2012) showed that accumulated 87 cost of least-cost paths and effective resistance estimates from circuit theory (McRae et al. 2008) are more sensitive to changes in cost surface than least-cost paths measured as Euclidean distance. Most recently, Zeller et al. (2017) showed that current density measures from circuit theory are sensitive to landscape definition, including spatial grain, thematic resolution, and the number of geospatial layers. Varying these aspects of landscape definition can alter the spatial 92 pattern of current density.

93 Circuit theory is used to measure landscape connectivity via the analogous relationship 94 between electricity travelling through a circuit and a random walk (Doyle \& Snell 1984). The 95 circuit theory model has increased in popularity in recent years, following the development of 96 the Circuitscape software package (McRae et al. 2008). Circuit theory differs from least-cost 97 path analysis in that multiple paths from a destination to a source are modeled rather than the 98 single, least-cost route, and the probability of moving through any pixel on a map is estimated.

99 This method may be preferable in cases where the assumption of travelling on the least-cost path 
100 is difficult to meet (Adriaensen et al. 2003), such as animals requiring knowledge of the optimal

101 route (Marrotte \& Bowman 2017). For example, gene flow is often modelled using circuit theory

102 (McRae \& Beier 2007).

103 Circuit theory analyses provide two outputs of particular interest for connectivity studies.

104 The first output is effective resistance, which is a pairwise measure of cost distance between

105 nodes and is measured as the voltage induced by passing 1 amp current between nodes (Doyle \& 106 Snell 1984, McRae et al. 2008). This is equivalent to commute time, or the expected time needed 107 to get from source to destination and back again. Effective resistance is often used to model 108 genetic connectivity between individuals or populations (e.g., McRae \& Beier 2007, Lee-Yaw et 109 al. 2009, Spear et al. 2010), but is not a mappable quantity. The second output, current density, is 110 a localized measure of movement probability at a spatially-referenced point that can be 111 interpreted as the probability of a location being used during a random walk between a source 112 and destination node. Current density can be mapped and is thus particularly useful for 113 applications where mapped routes are of interest, such as in natural heritage planning (Koen et 114 al. 2014), modelling animal movement routes (Walpole et al. 2012, Marrotte et al. 2020), or 115 forecasting migration arising from shifts in climate (Lawler et al. 2013). Recently, Marrotte et al. 116 (2017) used current density to estimate at-site genetic connectivity of sampled nodes.

117 Multiple sources of uncertainty exist when developing a cost surface to estimate current 118 density, including uncertainty about relative cost weights of different landscape elements, and 119 the thematic resolution (i.e., number of classes) of the cost surface map. Uncertainty in assigning 120 costs has been identified as a difficult and often subjective problem in modeling connectivity 121 (Peterman 2018, Grafius et al. 2017, Etherington 2016). Variability in the relative cost weights 122 assigned to particular landscape elements might affect current density, and since absolute costs 
123 are often unknown, the sensitivity of current density estimates to this variation is an important

124 consideration. If current density estimates are highly sensitive to such variation, then a priority

125 must be placed by users on accurate estimation of relative or absolute costs. On the other hand, if

126 current density is insensitive to variation in relative cost weights, then current estimates should

127 be robust to uncertainty. Beier, Majka, \& Newell (2009) showed that, provided rank order is

128 correct, least-cost route placement will be correct, but we are unaware of similar analysis

129 evaluating current density. A contrast to the rank order of costs being correct occurs where the

130 spatial arrangement of costs is varied, while other aspects of the cost surface are maintained.

131 Varying spatial arrangement of costs can influence the placement of a least-cost route (Rayfield

132 et al. 2009), and we anticipate a similar effect for circuit theory analysis. There might also be an

133 influence of the range of relative cost weights on current density estimates. Beier et al. (2009)

134 referred to this concept as dispersed versus compressed cost values, finding that least-cost path

135 analysis was robust to this type of uncertainty. Finally, landscape definition, including spatial

136 and thematic resolution, and the number of geospatial data layers employed, can affect estimates

137 of current density. Zeller et al. (2017) found that varying these characteristics of landscape

138 models produced different estimates of where the highest current density pixels occurred.

139 We evaluated the effects of varying relative cost weights, the range of cost weights, the

140 spatial arrangement of cost classes, and thematic resolution on current density estimates from

141 circuit theory. We predicted that, as with least-cost paths (Beier et al. 2009), current density

142 would not be sensitive to relative cost weights or the range of cost weights provided that the rank

143 order of the costs was not changed. We further predicted that changing the spatial arrangement of

144 relative cost weights would affect current density. Finally, we predicted that current density 
145 would not be sensitive to thematic resolution if the rank order of costs was maintained across 146 themes.

\section{Materials \& Methods}

149 We generated cost surface maps to test the sensitivity of current density estimates to changing cost values, range of cost values, varying spatial arrangement, and the number of cost classes (or thematic resolution). We started with eight cost surfaces using values from Rayfield et al. (2009), who selected cost scenarios that represented the range of values used in connectivity studies. We generated eight cost surface scenarios, each with three levels (low, medium, and high cost; Table 1). We also generated two additional scenarios using alternative low-cost values $(0.1,10)$ that were not used by Rayfield et al. (2009). For all simulated maps, we assigned $25 \%$ of cells low cost values, $25 \%$ medium cost values, and $50 \%$ high cost values.

We first used these 10 cost scenarios to evaluate the effects of relative cost weight and the range of cost weights on current density estimates. We did this at three different levels of fragmentation, high, medium, and low (Fig. 1). In the highly fragmented treatment, each pixel was randomly assigned to one of the three cost values. We produced 10 different high fragmentation maps - one for each cost scenario. Medium fragmentation maps were produced by placing 12 blocks of contiguous cost values within each map (10 maps in total), and low

163 fragmentation maps had 3 contiguous blocks (again, 10 maps in total) (Fig. 1). We used

164 Spearman rank correlations to compare current density values across all 10 cost scenarios within

165 fragmentation levels. As a contrast to varying the relative cost weights, we used the same 10 166 scenarios and 3 fragmentation levels to explore the effects of changing spatial arrangement of 167 cost classes on current density estimates. Changing spatial arrangement of costs is one form of 
168 rearranging cost ranks, allowing us to compare different configurations of cost surfaces with the

169 same overall costs and proportions of classes (50\% high, 25\% medium, and 25\% low). For this

170 analysis we used Spearman rank correlations to compare current density values between

171 fragmentation levels.

172 The range of cost values on a map might affect current density estimates by compressing

173 or dispersing the costs (Beier et al. 2009). If this is the case, the effects might be caused by the

174 high cost classes, since low costs are bounded by 0 in our design, and high costs have no such

175 bound (Koen et al. 2012). To test this idea, we evaluated the effect of the absolute value of the

176 difference in the range of costs between scenarios on the Spearman correlation between

177 scenarios for the simulated landscapes (Table 1). We also tested for a relationship between the

178 maximum current density from each scenario and the range of costs. We tested for this

179 relationship only within each fragmentation level (high, medium, and low).

180 We also sought to investigate our questions related to the effect of relative cost weights

181 and the range of cost weights on a real-world landscape, so we tested the effects of varying cost

182 weights on current density estimates in a $107-\mathrm{km}^{2}$ urban study area encompassing Oakville,

183 Ontario. The area was a highly fragmented urban landscape, consisting of $10 \%$ low, $14 \%$

184 medium and 76\% high cost values. Designation of cost values for the urban study area followed

185 the methodology of Bowman \& Cordes (2015) using the Southern Ontario Land Resource

186 Information System (SOLRIS) database (Ontario Ministry of Natural Resources 2008). We

187 varied costs in the urban landscape using the same 10 cost structures employed in the simulated

188 landscapes, producing 10 different maps. The resolution of the map was $15 \mathrm{~m}$.

189 We created an additional 20 simulated maps to test the effect of thematic resolution $(3,6,12$

190 cost classes per map) on current density (Fig. 2). We started with the low fragmentation scenario 
191 with the 3 thematic categories that we had already created. To generate 6 cost categories, we

192 divided the original three cost values from the low fragmentation map into portions reflecting $19345 \%$ and $55 \%$ of the costs per theme. These 6 subcategories were then divided by $48 \%$ and $52 \%$

194 to further stratify the cost surface map into 12 cost categories (Table 2). These proportions were 195 selected to maintain the rank order of the original 3 class map, while also varying thematic 196 resolution. We then compared outputs across these 3 different scenarios (3, 6, or 12 categories). 197 All simulated maps were $140 \times 140$ cells, consisting of $100 \times 100$ cells with a 20 -cell buffer, 198 to accommodate edge effects (Koen et al. 2010) on the nodes. Koen et al. (2014) showed that a 199 buffer equal to $20 \%$ of the width of the study area would largely remove the effect of node bias 200 in circuit analyses. The urban study area was comprised of 476,527 cells with an additional 201726,423 cells to accommodate the required buffer equal to $20 \%$ the width of the area. 202 We used Circuitscape v4.0 (McRae et al. 2013) to construct a current density map by 203 simulating electrical current flow iterated between all pairs of nodes on a rasterized surface. We 204 connected the eight neighbouring cells as an average cost using the pairwise mode and extracted 205 the cumulative current density map. The current density within a pixel, as a result of simulated 206 electrical current flowing across the surface is proportional to the probability of animal 207 movement (McRae et al. 2008). To model omnidirectional movement in the simulated 208 landscapes, we distributed 20 nodes in equal numbers among four sides of the surface in the 20209 cell buffer, using the same stratified placement of nodes for all scenarios. Due to the increased 210 size and more complex shape of the Oakville study area, 100 nodes were distributed in a 211 stratified placement around the buffered cost surface. See Marrotte et al. (2017), Bowman \& 212 Cordes (2015) and Koen et al. (2014) for additional descriptions of the point-based 213 omnidirectional methodology used. 
214 We note that circuit theory users will most often be interested in relative, rather than

215 absolute differences in current density. In fact, current density outputs are often standardized for 216 ease of comparison across landscapes (e.g., Bowman and Cordes 2015). Therefore, our method

217 of comparing current density outputs was aimed at evaluating relative differences in the

218 distribution of current across maps. In ArcMap v10.5 (ESRI 2016), we produced a polygon over 219 the generated study area and randomly selected 1000 data points within this polygon. We then 220 measured the correlation of the current density values of these randomly selected data points 221 across all maps by creating a Spearman rank correlation coefficient matrix in R v3.4.1 (R Core 222 Team 2014). We compared the mean correlation coefficients and the range of correlations across 223 scenarios of interest.

\section{Results}

226 In our simulated landscapes, the three fragmentation levels had equivalent costs for any given 227 cost scenario, varying only in the spatial arrangement of these costs. Mean (range) current 228 density for each of the three levels was 1.36 amperes for high fragmentation $(1.25$ to $1.59 ; \mathrm{n}=10$ 229 scenarios), 1.37 amperes for medium fragmentation (1.27 to 1.49), and 1.0 ampere for low 230 fragmentation (0.54 to 1.25$)$. The 6 and 12 class surfaces had mean values of 1.20 amperes (1.11 231 to 1.26 ) and 1.21 amperes (1.13 to 1.26$)$, respectively. The urban landscape had a mean (range) 232 current density of 3.95 amperes ( 3.78 to $4.31 ; \mathrm{n}=10$ scenarios).

234 across cost scenarios (Table 1; Fig. 3). High, medium, and low fragmentation levels had mean 235 Spearman correlation coefficients (range) of $0.87(0.65,1.00), 0.90(0.80,1.00)$, and $0.90(0.74$, $2361.00)(n=45$ comparisons for each analysis), respectively. Similarly, the urban study area (Fig. 
237 4) had a mean Spearman correlation coefficient (range) of $0.85(0.62,1.00)(n=45$

238 comparisons).

239

When the rank order of costs was not maintained, by changing the spatial arrangement of

240 the costs, correlations were reduced. The correlation coefficients for comparisons between

241 fragmentation levels (range) were -0.03 $(-0.17,0.06)$ for the comparison of low and medium

242 fragmentation, $-0.07(-0.10,-0.04)$ for medium and high, and $-0.02(-0.12,0.10)$ for low and high

243 levels of fragmentation ( $\mathrm{n}=100$ for each comparison) (Fig 3).

244 In simulated landscapes, the correlation between current density values was affected by

245 the range of cost values (Fig. 5). In general, correlations were more variable when the range of

246 cost values was broader, although, correlations always exceeded $R_{S}=0.65$. A broad range of

247 cost values occurred when the highest cost class was especially high, and this led to the

248 occurrence of especially high current density values (Fig. 6). Maximum current density in a

249 simulated landscape was correlated with cost range: $\mathrm{R}_{\mathrm{S}}=0.85,0.80$, and 0.57 for high, medium,

250 and low fragmentation levels, respectively ( $\mathrm{n}=10$ for each comparison). High current density

251 was associated with especially high cost classes, leading to overall high variability in current

252 density, reducing correlations with other cost scenarios. Maximum current density in the urban

253 landscape was also correlated with cost range $\left(\mathrm{R}_{\mathrm{S}}=0.78, \mathrm{n}=10\right.$ scenarios; Fig. 6$)$.

254 Within scenarios with 6 or 12 thematic classes, current density was highly correlated

255 across cost values: $R_{S}($ range $)=0.90(0.74,1.00)$ and $0.92(0.80,1.00)$, for 6 and 12 cost classes,

256 respectively ( $\mathrm{n}=45$ comparisons for each analysis). Comparing scenarios with different thematic

257 resolution, the Spearman correlation coefficients (range) were $0.87(0.69,0.97)$ for 3 and 6

258 classes, $0.89(0.75,0.98)$ for 6 and 12 , and $0.85(0.71,0.97)$ for 3 and $12(n=100$ for each

259 analysis) (Fig. 7). 


\section{Discussion}

261 We applied circuit theory to measure the sensitivity of current density estimates to varying cost 262 surfaces, including the relative cost weights, the range of cost weights, the spatial arrangement of 263 the classes throughout the landscape (fragmentation), and the number of classes (thematic 264 resolution). Given that the placement of least cost routes is robust to uncertainty in relative cost 265 weights and the range of costs (Beier, Majka, \& Newell 2009), we suspected that varying cost 266 weights would not substantially alter current density patterns from circuit theory when relative 267 ranks of costs were held constant. For a given level of fragmentation, current density was highly 268 correlated across landscape models despite differences in cost values. Thus, relative current 269 density estimates were not sensitive to cost value. This was true both for simulated landscapes 270 and a real-world urban landscape. The range of cost values had some effect on current density 271 however, as there was an inverse relationship between the ranges of cost values in a landscape 272 and the amount of correlation in current density of landscapes. We did find that changing the 273 spatial arrangement of cost classes had a high impact on current density, as shown by the low 274 correlation between different levels of fragmentation in our maps. Current density is sensitive to 275 landscape configuration. However, high correlations between current density estimates arising 276 from scenarios with varying numbers of classes suggests that thematic resolution itself has little 277 impact on current density, provided that the rank order of the costs is maintained across themes. 278 Our finding of low sensitivity to varying relative cost weights in circuit theory concurs with Beier et al. (2009)'s finding with least cost paths, in that provided the costs had a consistent 280 rank order, the current density maps were quite similar. This is a useful result, insofar as true 281 costs are often unknown by users (Grafius et al. 2017, Bishop-Taylor et al. 2015, Yumnam et al.

282 2014). Therefore, where users have insight about the relative costs of elements in the landscape, 
283 current density outputs should be reliable. This is helpful for applications where current density

284 is employed in natural heritage planning or designing wildlife linkages (e.g., Marrotte et al.

285 2017, Bowman \& Cordes 2015).

286 The range of cost values used in a map did affect the correlation between current density

287 values. As the range increased, current tended to be more highly pinched, whereas it spread more

288 as the range decreased (e.g., Fig. 3). This observation is further supported by the overall increase

289 in the variation of correlation results as the absolute cost value range increased (Fig. 5). The cost

290 range influenced current density through the effect of high cost weights. In our simulations, cost

291 was limited by 0 at the lower end, but was variable across scenarios at the upper end (e.g., Koen

292 et al. 2012). It was surfaces with the highest cost that also had the broadest range of costs (i.e. the

293 costs were more dispersed), and these surfaces produced higher current density values than

294 comparable surfaces with a narrower (i.e., compressed) cost ranges (Fig. 6). We are reminded

295 with this observation that high current density is a result of high cost weights (e.g., Marrotte et

296 al. 2017). Despite variation in current density values however, the Spearman correlations

297 between landscapes were always rather high within the fragmentation scenarios, such that there

298 was always agreement among maps, even where the range of cost values was high. The lowest

299 correlation between any pair of maps within fragmentation levels was $R_{S}=0.65$.

300 Our findings also concur with those of Rayfield et al. (2009), who found that the location

301 of least-cost paths varied with fragmentation. In particular, they showed that routes were more

302 sensitive to higher amounts of fragmentation, or lower quality matrix. We found that altering the

303 configuration of the landscape, while holding overall costs constant, affected the distribution of

304 current density. We consider that the low correlation across fragmentation scenarios was due to

305 the changing spatial arrangement of landscape elements, which altered the location of low and 
306 high cost routes. Changing the spatial arrangement served to change the rank order of the cost

307 categories, so this treatment served to show the effect of altering rank order.

We add to the work of Zeller et al. (2017), who found that current density estimates were

309

310

311

312

313

314

315

sensitive to landscape definition, which they varied by changing the data source, thematic resolution, and spatial grain. We did not explore the effect of spatial grain in our study, but Koen et al. (2019) have recently shown that reducing tile size can reduce accuracy of current density maps compared to untiled maps of larger extent. Our findings suggest that current density estimates are not particularly sensitive to thematic resolution, or relative cost weights, provided that the rank order of the costs does not vary. Coarsening of thematic resolution reduces detail, as similar categories are merged, but this can come with the potential benefit of increasing accuracy of ranking cost weights of the categories. More categories likely lead to increased error in ranking costs. Therefore, we recommend that users strike a balance between adequate detail and accurately ranked costs.

Our results suggest that current density is an effective tool for conservation planning, as it is robust to uncertainty in absolute cost values and sensitive to habitat fragmentation.

Fragmentation is an increasing conservation concern as the human footprint continues to expand; a recent global analysis revealed animal movements are reduced by approximately $50 \%$ in areas with a high human footprint (Tucker et al. 2018). Thus, understanding the movement of animals in and across complex landscapes will be imperative for effective conservation of biodiversity in the age of the Anthropocene. Our findings also suggest that when planning landscape connectivity applications, managers and researchers should put more effort toward establishing confidence in the rank order of costs, than reducing uncertainty in relative or absolute cost weights. 


\section{Conclusions}

331 We tested the hypothesis that current density estimates from circuit theory are not sensitive to

332 absolute values of underlying cost surfaces, provided that the rank order of the costs is accurate.

333 We compared a variety of cost surfaces with different costs, where rank order of costs was held

334 constant, and found a high correlation among resulting current density estimates. Our findings

335 suggest that knowledge of the absolute values of cost surfaces may not be required, provided that

336 rank orders of costs are known. This finding should facilitate the use of cost surfaces by

337 conservation practitioners interested in estimating connectivity and planning linkages and 338 corridors.

339

340 Acknowledgements

341 We thank Dr. Erin L. Koen for providing advice on an earlier version of the manuscript, and

342 Erica Newton for assistance with data.

343

344 References

345 Adriaensen, F., Chardon, J. P., De Blust, G., Swinnen, E., Villalba, S., Gulinck, H., \&

346 Matthysen, E. (2003) The application of 'least-cost' modelling as a functional landscape 347 model. Landscape and Urban Planning, 64, 233-247.

348 Beier, P., Majka, D. R., \& Newell, S. L. (2009) Uncertainty analysis of least-cost wildlife 349 modeling for designing linkages. Ecological Applications, 19, 2067-2077. 
350 Bishop-Taylor, R., Tulbure, M. G., \& Broich, M. (2015) Surface water network structure,

351 landscape resistance to movement and flooding vital for maintaining ecological connectivity

352 across Australia's largest river basin. Landscape Ecology, 30, 2045-2065.

353 Bowman, J., \& Cordes, C. (2015). Landscape connectivity in the Great Lakes basin. Figshare.

$354 \quad 10.6084 / \mathrm{m} 9$. figshare.1471658.v1.

355 Crooks, K. R., Burdett, C.L., Theobald, D. M., King, S. R. B., Marco, M. D., Rondinini,

356 C., \& Boitani, L. (2017), Quantification of habitat fragmentation reveals extinction risk in

357 terrestrial mammals. Proceedings of the National Academy of Sciences of the United States

358 of America, 114(29), 7635-7640.

359 Cushman, S. A., McKelvey, K. S., Hayden, J., \&Schwartz M. K. (2006) Gene flow in complex

360 landscapes: testing multiple hypotheses with causal modeling. The American Naturalist, $361 \quad 168(4), 486-499$.

362 Doyle P. G., \& Snell J.L. (1984) Random walks and electric networks. Washington:

363 Mathematical Association of America. 159 p.

364 ESRI (2016) ArcGIS Desktop: Release 10.5. Redlands, CA: Environmental Systems Research $365 \quad$ Institute.

366 Etherington, T.R. (2016) Least-cost modelling and landscape ecology: concepts, applications, 367 and opportunities. Current Landscape Ecology Reports, 1, 40-53.

368 Grafius, D. R., Corstanje, R., Siriwardena, G. M., Plummer, K. E., \& Harris, J. A. (2017) A

369 bird's eye view: using circuit theory to study urban landscape connectivity for birds.

$370 \quad$ Landscape Ecology, 32, 1771-1787.

371 Gu, W., Heikkilä, R., \& Hanski, I. (2002) Estimating the consequences of habitat fragmentation 372 on extinction risk in dynamic landscapes. Landscape Ecology, 17, 699-710. 
373 Gubbi S., Mukherjee K., Swaminath M. H, \& Poornesha H. C. (2015) Providing more protected 374 space for tigers Panthera tigris: a landscape conservation approach in the Western Ghats, 375 southern India. Oryx, 50, 336-43.

376 Koen, E. L., Bowman, J., Sadowski, C., \& Walpole, A. A. (2014) Landscape connectivity for 377 wildlife: development and validation of multispecies linkage maps. Methods in Ecology and $378 \quad$ Evolution, 5, 626-633.

379 Koen, E. L., Bowman, J., \& Walpole, A. A. (2012) The effect of cost surface parameterization 380 on landscape resistance estimates. Molecular Ecology Resources 12, 686-696.

381 Koen, E. L., E. H. Ellington, \& Bowman J. (2019) Mapping landscape connectivity for large $382 \quad$ spatial extents. Landscape Ecology 34, 2421-2433.

383 Koen, E. L., Garroway, C. J., Wilson, P. J., \& Bowman, J. (2010) The effect of map boundary on 384 estimates of landscape resistance to animal movement. PLoS ONE, 5(7), e11785.

Lawler, J. J., Ruesch, A. S., Olden, J. D., \& McRae, B. H. (2013) Projected climate-driven faunal 386 movement routes. Ecology Letters, 16, 1014-1022.

387 388 389
Lee-Yaw, J. A., Davidson, A., McRae, B. H., \& Green, D. M. (2009) Do landscape processes predict phylogeographic patterns in the wood frog? Molecular Ecology, 18, 1863-1874.

Marrotte, R. R., Bowman, J., Brown, M. G., Cordes, C., Morris, K. Y., Prentice, M. B., \& Wilson, P. J. (2017) Multi-species genetic connectivity in a terrestrial habitat network. Movement Ecology, 5(1), 21.

Marrotte, R. R., Bowman, J., \& Wilson, P.J. (2020) Climate connectivity of the bobcat in the Great Lakes Region. Ecology and Evolution doi.org/10.1002/ece3.6049.

McRae, B.H. (2006) Isolation by resistance. Evolution, 60,1551-1561. 
395 McRae, B. H., \& Beier, P. (2007) Circuit theory predicts gene flow in plant and animal 396 populations. Proceedings of the National Academy of Sciences, 104, 19885-19890.

397 McRae, B. H., Dickson, B. G., Keitt, T. H., \& Shah, V. B. (2008) Using circuit theory to model 398 connectivity in ecology, evolution, and conservation. Ecology, 89, 2712-2724.

399 McRae, B. H., Shah, V. B., \& Mohapatra, T. K. (2013) Circuitscape 4 User Guide. The Nature 400 Conservancy. http://www.circuitscape.org.

401 Peterman, W. E. (2018) ResistanceGA: An R package for the optimization of resistance surfaces 402 using genetic algorithms. Methods in Ecology and Evolution, 9: 1638-1647.

403 R Core Team (2014). R: A language and environment for statistical computing. R Foundation for $404 \quad$ Statistical Computing, Vienna, Austria.

405 http://www.R-project.org/.

406 Rayfield, B., Fortin, M. J., \& Fall, A. (2010) The sensitivity of least-cost habitat graphs to 407 relative cost surface values. Landscape Ecology, 25, 519-532.

408 Spear, S. F., Balkenhol, N., Fortin, M. J., McRae, B. H., \& Scribner, K. I. M. (2010) Use of 409 resistance surfaces for landscape genetic studies: considerations for parameterization and $410 \quad$ analysis. Molecular Ecology, 19, 3576-3591.

411 Taylor, P. D., Fahrig, L., Henein, K., \& Merriam, G. (1993) Connectivity is a vital element of $412 \quad$ landscape structure. Oikos, 68, 571-573.

413 Tucker, M.A., Böhning-Gaese, K., Fagan, W.F., Fryxell, J.M., Van Moorter, B., Alberts, S.C., 414 Ali, A.H., Allen, A.M., Attias, N., Avgar, T. \& Bartlam-Brooks, H. (2018) Moving in the 415 Anthropocene: Global reductions in terrestrial mammalian movements. Science, 359, 466416469. 
417 Walpole, A. A., Bowman, J., Murray, D. L., \& Wilson, P. J. (2012) Functional connectivity of 418 lynx at their southern range periphery in Ontario, Canada. Landscape Ecology, 27, 761-773.

419 Yumnam B., Jhala Y. V., Qureshi Q., Maldonado J. E., Gopal R., Saini S., Srinivas, Y., \&

420 Fleischer, R. C. (2014) Prioritizing tiger conservation through landscape genetics and habitat $421 \quad$ linkages. PLoS ONE, 9(11), e111207.

422 Zeller, K. A., McGarigal, K., Cushman, S. A., Beier, P., Vickers, T. W. Boyce, W. M. (2017)

423 Sensitivity of resource selection and connectivity models to landscape definition. Landscape $424 \quad$ Ecology, 32, 835-855.

425 


\section{Table $\mathbf{1}$ (on next page)}

Cost scenarios used to compare across simulated and urban landscapes with 3 cost categories. 
Table 1. Cost values assigned to each cost category (low, medium, high).

\begin{tabular}{|c|c|c|c|c|}
\hline Scenario & Low & Medium & High & Range \\
\hline C1 & 0.1 & 0.5 & 1 & 0.99 \\
\hline $\mathrm{C} 2$ & 1 & 1.5 & 2.25 & 1.25 \\
\hline C3 & 1 & 1.5 & 150 & 149 \\
\hline C4 & 1 & 2 & 3 & 2 \\
\hline C5 & 1 & 2 & 200 & 199 \\
\hline C6 & 1 & 5 & 7.5 & 6.5 \\
\hline C7 & 1 & 5 & 500 & 499 \\
\hline C8 & 1 & 100 & 150 & 149 \\
\hline C9 & 1 & 100 & 10000 & 9999 \\
\hline C10 & 10 & 100 & 1000 & 990 \\
\hline
\end{tabular}




\section{Table 2 (on next page)}

Cost scenarios used to compare across 6 and 12 category thematic resolutions while maintain rank order of costs in comparison to a 3 category cost surface. 
Table 2. Cost values assigned to each category for thematic resolutions with 6 and 12 categories.

\begin{tabular}{|c|c|c|c|c|c|c|c|c|c|c|c|c|c|c|c|c|c|c|}
\hline \multirow[b]{3}{*}{ C1 } & \multicolumn{6}{|c|}{ Six categories } & \multicolumn{12}{|c|}{ Twelve Categories } \\
\hline & \multicolumn{2}{|c|}{ Lower } & \multicolumn{2}{|c|}{ Medium } & \multicolumn{2}{|c|}{ Higher } & \multicolumn{4}{|c|}{ Lower } & \multicolumn{4}{|c|}{ Medium } & \multicolumn{4}{|c|}{ Higher } \\
\hline & 0.045 & 0.055 & 0.225 & 0.275 & 0.45 & 0.55 & 0.0216 & 0.0234 & 0.0264 & 0.0286 & 0.108 & 0.117 & 0.132 & 0.143 & 0.216 & 0.234 & 0.264 & 0.286 \\
\hline C2 & 0.45 & 0.55 & 0.675 & 0.825 & 1.0125 & 1.2375 & 0.216 & 0.234 & 0.264 & 0.286 & 0.324 & 0.351 & 0.396 & 0.429 & 0.486 & 0.5265 & 0.594 & 0.6435 \\
\hline C3 & 0.45 & 0.55 & 0.675 & 0.825 & 67.5 & 82.5 & 0.216 & 0.234 & 0.264 & 0.286 & 0.324 & 0.351 & 0.396 & 0.429 & 32.4 & 35.1 & 39.6 & 42.9 \\
\hline C4 & 0.45 & 0.55 & 0.9 & 1.1 & 1.35 & 1.65 & 0.216 & 0.234 & 0.264 & 0.286 & 0.432 & 0.468 & 0.528 & 0.572 & 0.648 & 0.702 & 0.792 & 0.858 \\
\hline C5 & 0.45 & 0.55 & 0.9 & 1.1 & 90 & 110 & 0.216 & 0.234 & 0.264 & 0.286 & 0.432 & 0.468 & 0.528 & 0.572 & 43.2 & 46.8 & 52.8 & 57.2 \\
\hline C6 & 0.45 & 0.55 & 2.25 & 2.75 & 3.375 & 4.125 & 0.216 & 0.234 & 0.264 & 0.286 & 1.08 & 1.17 & 1.32 & 1.43 & 1.62 & 1.755 & 1.98 & 2.145 \\
\hline C7 & 0.45 & 0.55 & 2.25 & 2.75 & 225 & 275 & 0.216 & 0.234 & 0.264 & 0.286 & 1.08 & 1.17 & 1.32 & 1.43 & 108 & 117 & 132 & 143 \\
\hline C8 & 0.45 & 0.55 & 45 & 55 & 67.5 & 82.5 & 0.216 & 0.234 & 0.264 & 0.286 & 21.6 & 23.4 & 26.4 & 28.6 & 32.4 & 35.1 & 39.6 & 42.9 \\
\hline C9 & 0.45 & 0.55 & 45 & 55 & 4500 & 5500 & 0.216 & 0.234 & 0.264 & 0.286 & 21.6 & 23.4 & 26.4 & 28.6 & 2160 & 2340 & 2640 & 2860 \\
\hline C10 & 4.5 & 5.5 & 45 & 55 & 450 & 550 & 2.16 & 2.34 & 2.64 & 2.86 & 21.6 & 23.4 & 26.4 & 28.6 & 216 & 234 & 264 & 286 \\
\hline
\end{tabular}




\section{Figure 1}

Simulated cost surfaces with 3 levels of fragmentation.

Cost surfaces with $100 \times 100$ pixels and a 20-pixel buffer around the perimeter $(140 \times 140$ cells total). All maps had a total ratio of $25 \%$ low cost (white), $25 \%$ medium cost (grey), and $50 \%$ high cost (black). Treatments included (A) High fragmentation (randomized), (B) Medium fragmentation, and (C) Low fragmentation. Each cell was assigned a weight corresponding to its cost. For each level of fragmentation, we generated 10 maps with varying cost weights. 
A)

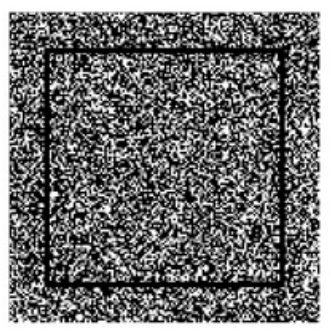

B)

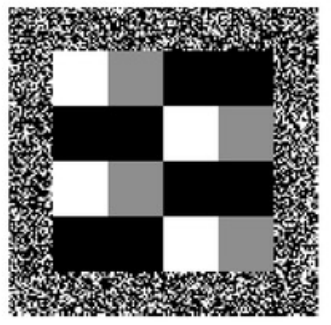

C)

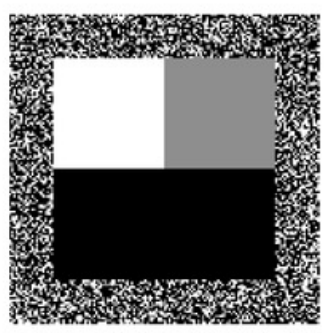




\section{Figure 2}

Simulated cost surfaces with varying thematic resolution $(3,6$, and 12 categories).

Cost surfaces with $100 \times 100$ pixels and a 20-pixel buffer around the perimeter $(140 \times 140$ cells total). All maps had a total ratio of $25 \%$ low cost (white), $25 \%$ medium cost (grey), and $50 \%$ high cost (black). Treatments included 3 levels of thematic resolution (A) 3 categories, (B) 6 categories, and (C) 12 categories. Each cell was assigned a weight corresponding to its cost. For each level of thematic resolution, we generated 10 maps with varying cost weights. 
A)

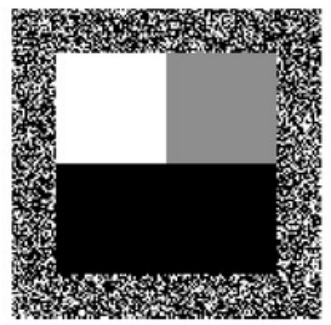

B)

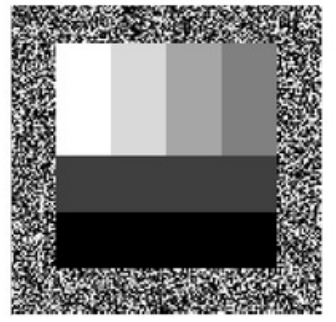

C)

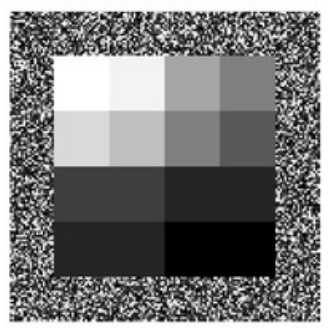




\section{Figure 3}

Example current density maps for cost surfaces at 3 levels of fragmentation.

Examples of current density maps at a resolution of $140 \times 140$ cells for landscapes with different fragmentation levels (high, medium, and low), different cost classes, and a 20-pixel buffer. Panels A, C and E had a costs of 7.5 (high), 5, and 1 (low), whereas B, D, and F had costs of 1000,100,10, respectively. Highest current density is indicated by red. Node locations are indicated by areas of high current density around perimeters, especially evident in panels $A$ and $F$. 
A)

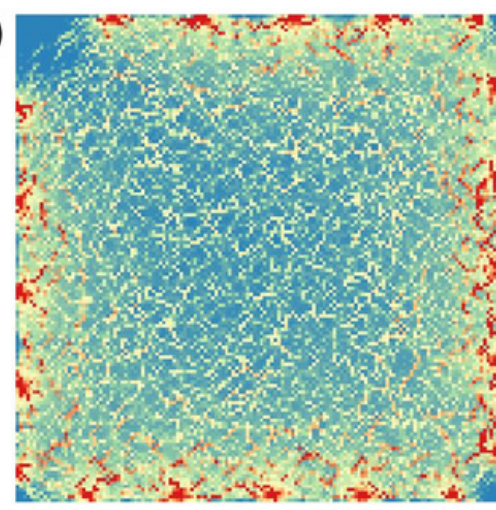

C)

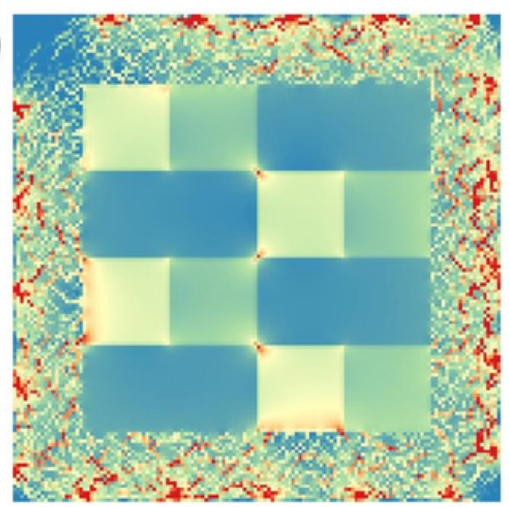

E)

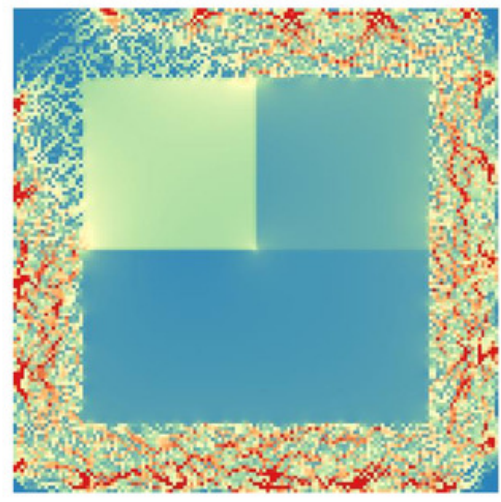

B)

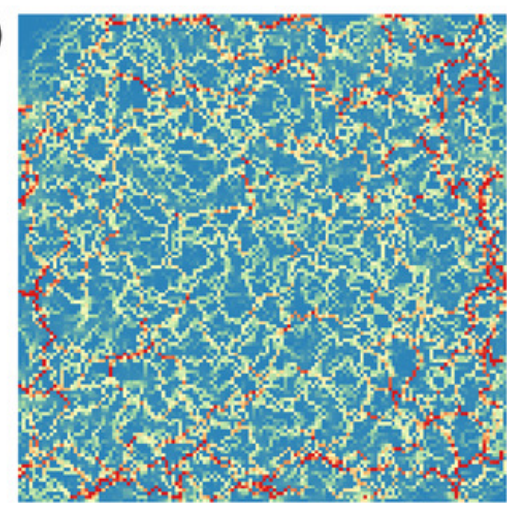

D)

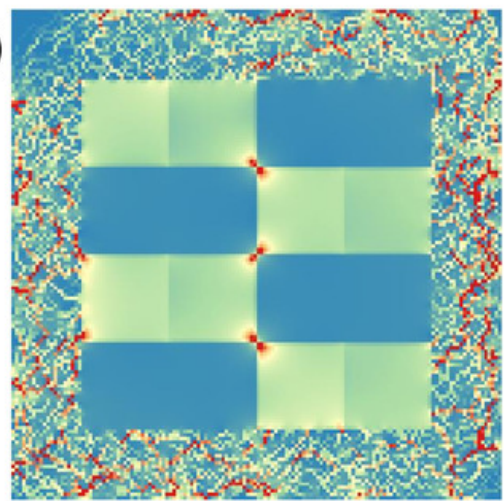

F)

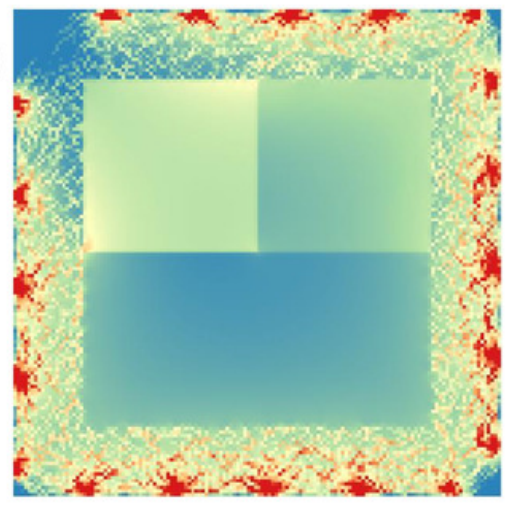




\section{Figure 4}

Example current density maps from an urban landscape.

Two examples of current density maps for an urban study area in Oakville, Ontario. Panel A had costs of 1 (low), 2, and 3 (high), and B had costs of 1, 100, and 150. The Spearman rank correlation was 0.68 , lowest of all comparisons for this landscape (mean (range) RS $=0.85$ (0.62-1.00) $n=45$ comparisons). 

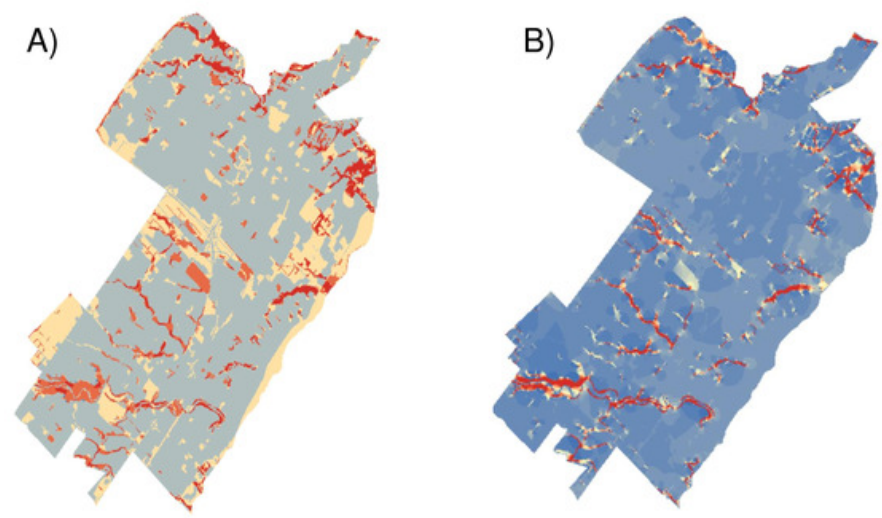


\section{Figure 5}

Effect of the range of cost values on correlation of current density across landscapes

Effect of the absolute value of the difference in the range of cost values in a landscape ( $\log _{10^{-}}$ transformed) on the Spearman correlation between current density estimates in pairs of landscapes: (A) high, (B) medium, and (C) low fragmentation. 
A)

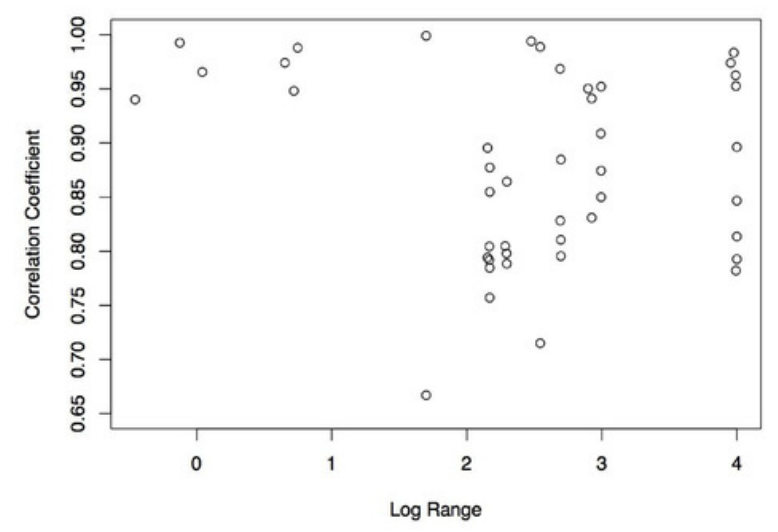

B)

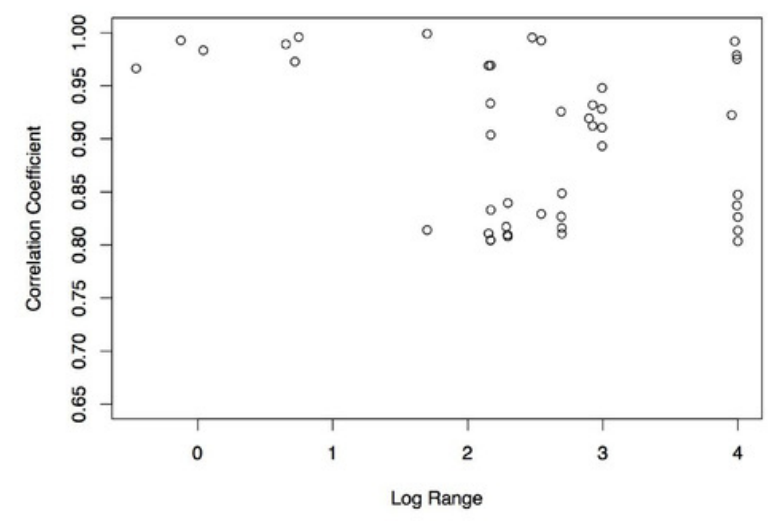

C)

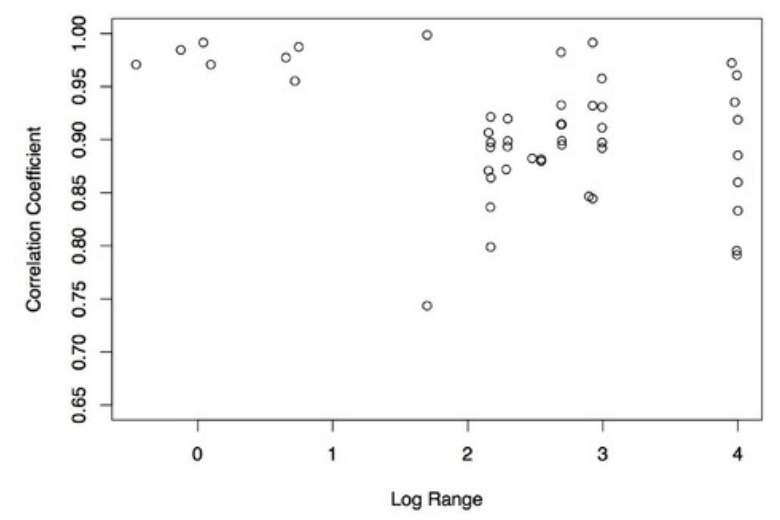


Figure 6

Effect of cost range on current density

Effect of the absolute value of the difference in the range of cost values in a landscape ( $\log _{10^{-}}$ transformed) on mean and maximum current density estimates cross 10 different cost scenarios. We have shown here (A) the medium fragmentation scenarios in a simulated landscape, and (B) the real, urban landscape. 
A)

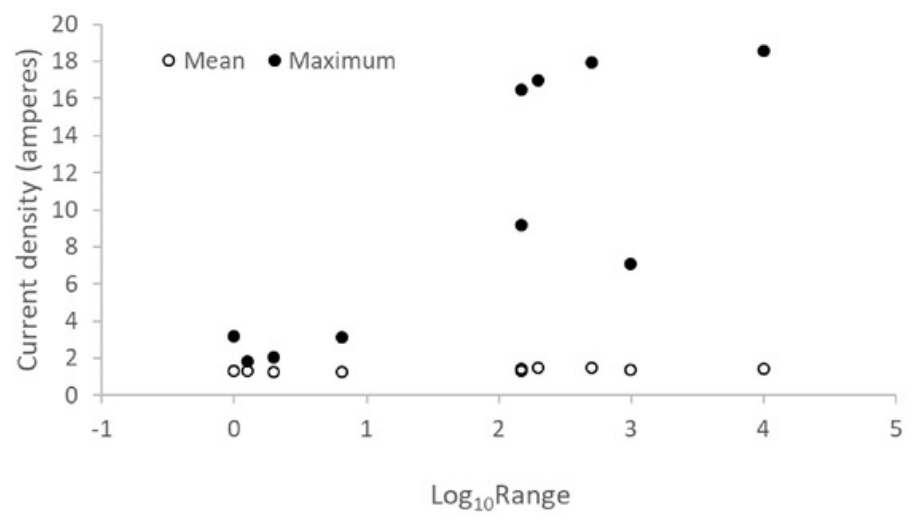

B)

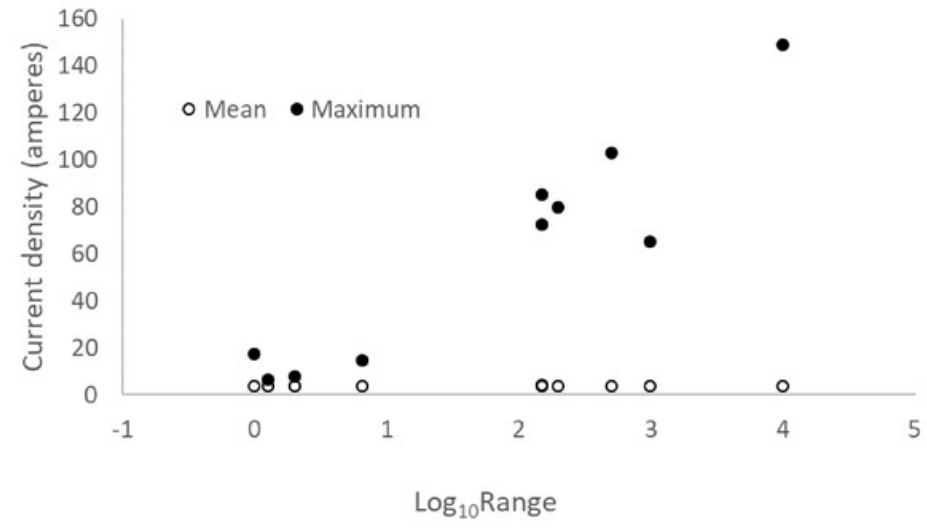




\section{Figure 7}

Examples of current density maps with different thematic resolutions

Figure 7. Examples of current density maps at a resolution of $140 \times 140$ cells for landscape depictions with different thematic resolutions (3 (top row), 6, or 12 (bottom row) categories), different costs, and a 20-pixel buffer. Panels A, C and E had costs of 1 (low), 5, and 7.5 (high), whereas $B, D$, and F had costs of $10,100,1000$, respectively. Highest current density is indicated by red. Node locations are indicated by areas of high current density around perimeters. 
A)

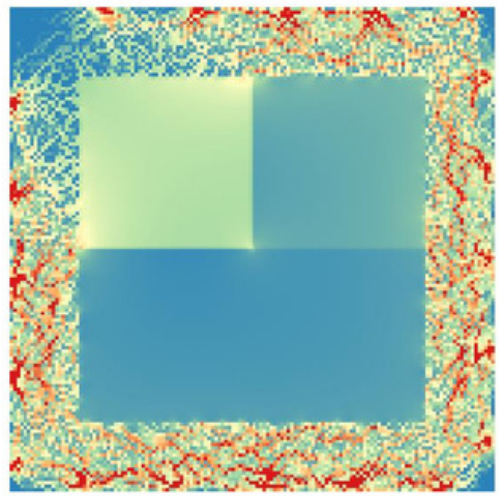

C)

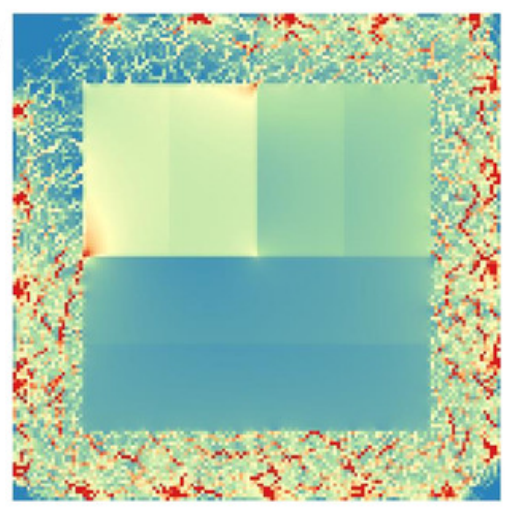

E)

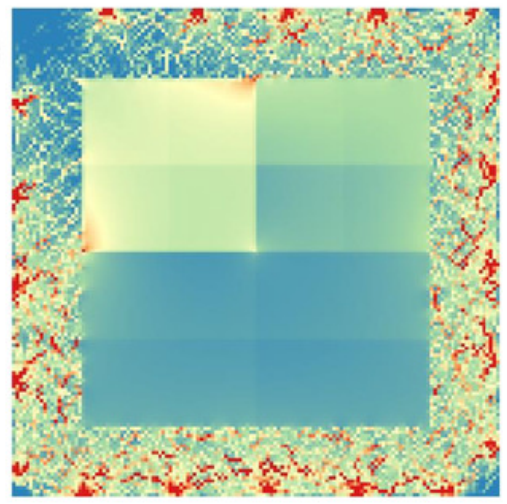

B)

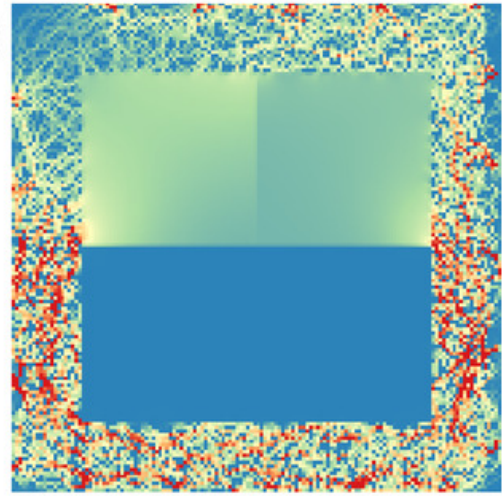

D)

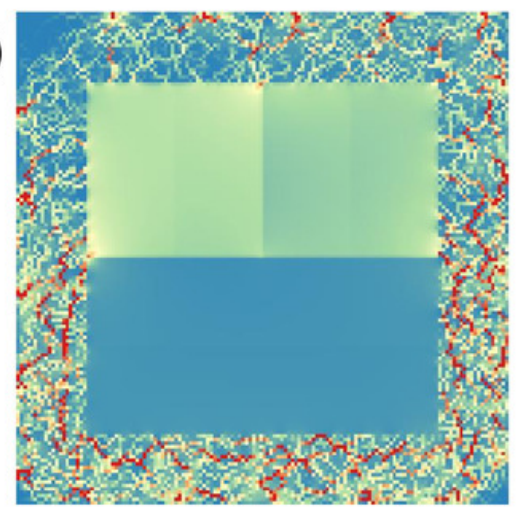

F)

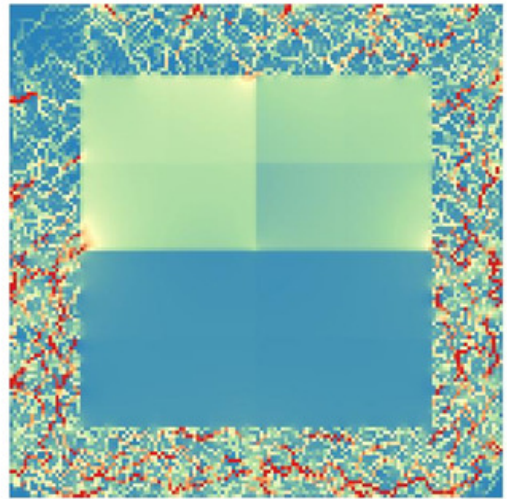

